\title{
La economía de la región Nordeste del Brasil a la luz de la matriz de insumo-producto regional de 2011
}

\author{
Marcos Falcão Gonçalves, Mateus de Carvalho Reis Neves \\ y Marcelo José Braga
}

\section{Resumen}

En este trabajo se busca determinar los sectores protagonistas de la economía de la región Nordeste del Brasil a la luz de la metodología de la matriz de insumo-producto, sobre la base de la estructura económica de 2011. Para ello, se actualizó la matriz de insumo-producto regional de 2004 a 2011 y, posteriormente, se calcularon los índices de encadenamiento de Rasmussen-Hirschman, el campo de influencia y los índices puros de encadenamiento, así como los multiplicadores de producción, empleo e ingreso, de tipos I y II. Los resultados ratifican la importancia de los sectores textil, de productos químicos y aquellos vinculados a la industria del petróleo e indican la producción de bienes intermedios como una de las características de la economía del Nordeste.

\section{Palabras clave}

Condiciones económicas, desarrollo económico, desarrollo regional, producción industrial, empleo, ingresos, análisis de insumo-producto, Brasil

\section{Clasificación JEL}

R10, R11, R13

\section{Autores}

Marcos Falcão Gonçalves es Coordinador General de Estudios e Investigación, Evaluación, Tecnología e Innovación de la Superintendencia de Desarrollo del Nordeste (SUDENE), Brasil. Correo electrónico: marcos.falcao@sudene.gov.br.

Mateus de Carvalho Reis Neves es Profesor del Departamento de Economía Rural (DER) de la Universidad Federal de Viçosa (UFV) - Minas Gerais, Brasil. Correo electrónico: mateus.neves@ufv.br.

Marcelo José Braga es Profesor del Departamento de Economía Rural (DER) de la Universidad Federal de Viçosa (UFV) - Minas Gerais, Brasil. Correo electrónico: mjbraga@ufv.br. 


\section{Introducción}

En la década de 1970 se intensificaron las iniciativas para descentralizar la economía brasileña por medio de inversiones públicas en infraestructura (carreteras, puertos, energía, entre otras cosas) y sectores industriales estratégicos, incluso durante el II Plano Nacional de Desenvolvimento (1975-1979) (Gobierno del Brasil, 1970)ํ. Como consecuencia de estas iniciativas, en el período de 1970 a 1984 se establecieron grandes complejos productivos en el Nordeste ${ }^{2}$ (Galindo, 1997).

El proceso de descentralización parece persistir en la actualidad, pues la participación en el valor de la transformación industrial de la región Sudeste se redujo del 69,3\% en 1996 al 61,6\% en 2004, mientras la de la región Nordeste aumentó del 4,3\% al 10,1\% (BCB, 2006). Esto subraya el crecimiento económico que se observa en la región, a pesar de las heterogeneidades que caracterizan este proceso, en el que algunas áreas dinámicas de desarrollo regional coexisten con áreas enteras estancadas y carentes de modernización productiva.

La matriz de insumo-producto se ha utilizado a menudo en el diseño o el análisis de políticas públicas. Su amplia utilización se justifica por su capacidad predictiva de los posibles efectos que un choque en la demanda final de determinado sector puede provocar, tanto en el propio sector como en el resto de la economía.

De esta forma, permite a los responsables de la planificación de políticas estimular los sectores considerados clave, es decir, los sectores en los que un choque en la demanda final se propaga con mayor intensidad al resto de la economía, tanto en las fases preliminares (hacia atrás), como ulteriores (hacia adelante) de la cadena productiva, así como encontrar y corregir los cuellos de botella productivos.

En este contexto, como observan Tosta, Lirio y Silveira (2004), los modelos de insumo-producto han contribuido a una amplia gama de trabajos y análisis económicos, pues permiten la verificación de las repercusiones en diferentes sectores a partir de los cambios en la producción de un sector específico.

Sin embargo, los trabajos sobre la economía del Nordeste basados en dicha metodología todavía son escasos. En uno de esos estudios, Guilhoto y otros (2012) presentan un mayor enfoque teórico y metodológico y construyen una matriz de insumo-producto regional (año base 2004), calculando los principales indicadores y adoptando también un corte estatal. Tomando como referencia el trabajo de Guilhoto y otros (2012), Ribeiro y otros (2013) analizaron la microrregión de Suape (en el estado de Pernambuco) y los efectos económicos potenciales de la fase de construcción de la refinería Abreu y Lima, considerada en el estudio como núcleo relevante de la industria motriz.

La matriz de insumo-producto también fue utilizada por Morrone (2017) para verificar la estructura básica de la economía de Rio Grande do Sul en 2008 y estimar el impacto del aumento del impuesto sobre la circulación de mercaderías y servicios (ICMS) en la demanda final y la actividad económica del estado. El estudio muestra el efecto negativo de la medida, que tiende a limitar las posibilidades de desarrollo regional.

Montoya, Finamore y Pasqual (2012) también utilizaron la matriz de insumo-producto para analizar las fuentes de crecimiento y el cambio estructural en la economía de Rio Grande do Sul en el período de 1998 a 2003. La matriz refleja la reducción y la reanudación del crecimiento económico.

\footnotetext{
1 Véanse más detalles sobre el II Plano Nacional de Desenvolvimento (1975-1979) y el contexto económico brasileño de esa época en Lessa (1977), Delgado (1985) y Fishlow (1986).

2 Se destacan el polo petroquímico de Camaçari, en Bahia; el polo integrado petrocloroquímico en Sergipe; el complejo cloroquímico y de sal gema en Alagoas; el complejo azucarero-alcoholero del litoral oriental de Pernambuco y Alagoas; la agroindustria del perímetro irrigado del río São Francisco medio de Pernambuco y Bahia; la industria portuaria de Suape, en Pernambuco; la industria química y metalúrgica de Rio Grande do Norte; la industria textil y de prendas de vestir de Ceará; la industria mineral-metalúrgica de Maranhão; y la explotación del petróleo a lo largo del litoral del Nordeste (Galindo, 1997).
} 
En forma análoga, Ribeiro y Leite (2012) realizaron un análisis con enfoque estatal y elaboraron una matriz de insumo-producto para el estado de Sergipe, por medio del "método RAS" para la construcción de matrices regionales ${ }^{3}$. Los autores señalaron que la economía de Sergipe presentaba problemas en la estructura de oferta de insumos en sectores cruciales para el desarrollo del estado pues, además de estar concentrada sectorialmente y presentar una baja inserción internacional, tenía pocos sectores clave que indujeran el crecimiento local.

Teniendo en cuenta las vicisitudes regionales, en este trabajo se planteó el objetivo de determinar los sectores que actualmente son protagonistas en la economía de la región Nordeste del Brasil, a la luz de la metodología de la matriz de insumo-producto, sobre la base de la estructura económica de 2011, estableciendo, además, puntos relevantes para la orientación de las políticas públicas dirigidas al desarrollo de la región. Para ello, se actualizó la matriz de insumo producto del Nordeste de 2004 a 2011 y, posteriormente, se calcularon los índices de encadenamiento de Rasmussen-Hirschman ${ }^{4}$, el campo de influencia ${ }^{5}$ y los índices puros de encadenamiento ${ }^{6}$, así como los multiplicadores de producción, empleo e ingreso, de tipos I y $\|^{7}$.

Para comprender mejor los instrumentos utilizados, en la segunda sección se presenta una breve referencia teórica relativa al modelo de insumo-producto. En la tercera sección se abordan los aspectos metodológicos aplicados en el trabajo, mientras en la cuarta se determinan los principales sectores de la economía de la región Nordeste. A continuación se presentan las consideraciones finales.

\section{Fundamentación teórica}

De acuerdo con Miller y Blair (2009), un modelo de insumo-producto consiste en un sistema de ecuaciones lineales, cada una de las cuales describe la distribución de los productos de un sector en toda la economía. Su objetivo fundamental es analizar la interdependencia de los sectores económicos. Guilhoto y otros (2012) comparan el modelo de insumo-producto propuesto por Leontief (1966) con una "fotografía económica" de la propia economía, que puede evidenciar la manera en que sus sectores se relacionan entre sí o, en otras palabras, determinar los sectores que demandan productos y servicios y aquellos que los ofrecen.

Los cuadros de insumo-producto suelen utilizarse para representar dicha interdependencia, que se sistematiza en el cuadro 1.

\footnotetext{
3 Véanse más detalles sobre el método RAS en Miller (1998, pág. 89).

4 Conforme Rasmussen (1956) y Hirschman (1958).

5 Conforme la sugerencia de Mendes, Pereira y Teixeira (2011).

6 Propuesto por Guilhoto y otros (1994).

7 Véanse más detalles en Miller y Blair (2009).
} 
Cuadro 1

Matriz de insumo-producto de Leontief para dos sectores

\begin{tabular}{|c|c|c|c|c|c|c|c|c|c|}
\hline \multirow{3}{*}{ Sectores } & \multicolumn{8}{|c|}{ Compras (j) } & \multirow{3}{*}{$\begin{array}{l}\text { Valor bruto de } \\
\text { la producción }\end{array}$} \\
\hline & \multicolumn{3}{|c|}{ Demanda intermedia } & \multicolumn{5}{|c|}{ Demanda final } & \\
\hline & Sector 1 & Sector 2 & Subtotal & $\mathrm{C}$ & 1 & G & $E$ & Subtotal & \\
\hline \multirow{2}{*}{ Ventas (i) } & $Z_{11}$ & $Z_{12}$ & $\sum_{j=1}^{2} z_{i j}$ & $C_{1}$ & $I_{1}$ & $G_{1}$ & $E_{1}$ & $Y_{1}$ & $X_{1}$ \\
\hline & $Z_{21}$ & $Z_{22}$ & $\sum_{j=1}^{2} z_{2 j}$ & $C_{2}$ & $I_{2}$ & $G_{2}$ & $E_{2}$ & $Y_{2}$ & $X_{2}$ \\
\hline Subtotal & $\sum_{i=1}^{2} z_{i 1}$ & $\sum_{i=1}^{2} z_{i 2}$ & $\sum_{i, j=1}^{2} z_{i j}$ & $\sum_{i=1}^{2} C_{i}$ & $\sum_{i=1}^{2} I_{i}$ & $\sum_{i=1}^{2} G_{i}$ & $\sum_{i=1}^{2} E_{i}$ & $\sum_{i=1}^{2} Y_{i}$ & $\sum_{i=1}^{2} X_{i}$ \\
\hline Importaciones & $M_{1}$ & $M_{2}$ & $\sum_{i=1}^{2} M_{j}$ & & & & & & \\
\hline Impuestos indirectos netos & $T_{1}$ & $T_{2}$ & $\sum_{i=1}^{2} T_{j}$ & & & & & & \\
\hline Valor agregado & $V A_{1}$ & $V A_{2}$ & $\sum_{i=1}^{2} V A_{j}$ & & & & & & \\
\hline Valor bruto de la producción & $X_{1}$ & $X_{2}$ & $\sum_{i=1}^{2} X_{j}$ & & & & & & \\
\hline
\end{tabular}

Fuente: Elaboración propia sobre la base de J. J. M. Guilhoto, Análise de insumo-produto: teoria, fundamentos e aplicações, São Paulo, Facultad de Economía, Administración y Contabilidad, Universidad de São Paulo (FEA/USP), 2007.

Donde $z_{i j}$ es la provisión de insumos del sector $i$ al sector $j$; $C_{i}$ es la provisión del sector $i$ destinada al consumo final privado; $I_{i}$ es la provisión del sector $i$ destinada a la inversión privada; $G_{i}$ es la provisión del sector $i$ destinada al gobierno (consumo e inversión); $E_{i}$ es la provisión del sector $i$ destinada a las exportaciones al resto del mundo; $Y_{i}$ se refiere a la demanda final total satisfecha por el sector $i$ $\left(C_{i}+I_{i}+G_{i}+E_{i}\right) ; X_{i}$ representa el valor bruto de la producción del sector $i$ (u oferta total de $i$ ); $M_{j}$ corresponde a las importaciones realizadas por el sector $j$; $T_{j}$ es el total de impuestos indirectos netos recaudados por el sector $j$; $V A_{j}$ es el valor agregado bruto total del sector $j$; y $C_{j}$ es el costo total de producción del sector $j$.

A partir del cuadro 1, se obtiene la ecuación de producción mediante la ecuación (1):

$$
X_{i}=\sum_{j=1}^{2} z_{i j}+Y_{i}
$$

Considerando los supuestos del modelo de Leontief, contenidos en Miller y Blair (2009), a saber: i) relaciones fijas entre los insumos de un sector y su producto y; ii) rendimientos constantes a escala, el coeficiente técnico de producción, también llamado coeficiente de insumo-producto o coeficiente de insumo directo, se deduce por medio de la ecuación (2):

$$
a_{i j}=\frac{z_{i j}}{x_{j}}
$$

donde $\alpha_{i j}$ es el coeficiente técnico que indica la cantidad de insumos del sector $i$ necesarios para la producción de una unidad de producto final en el sector $j$. 
Al aplicar (2) en (1) y generalizar a $n$ sectores, se obtiene la ecuación (3):

$$
X_{i}=\sum_{j=1}^{n} a_{i j} x_{j}+Y_{i}
$$

En forma matricial, la ecuación (3) puede escribirse como (4):

$$
X=A X+Y
$$

Dado que la demanda final es exógena, se deduce que:

$$
X-A X=Y
$$

o, también:

$$
(I-A)^{-1} Y=X
$$

donde el término $(I-A)^{-1}$, también denominado matriz $B$, corresponde a la matriz de coeficientes directos e indirectos, o matriz de Leontief. Esta matriz tiene una dimensión $n \times n$, en que $n$ indica el número de sectores considerados, cuyos elementos pueden representarse por $b_{i j}$.

A partir del modelo nacional, Miller y Blair (2009) propusieron un modelo regional, como se

\begin{tabular}{|c|c|c|c|c|c|c|}
\hline & & & \multicolumn{4}{|c|}{ Sectores compradores } \\
\hline & & & \multicolumn{2}{|c|}{ Región L } & \multicolumn{2}{|c|}{ Región M } \\
\hline & & & 1 & 2 & 1 & 2 \\
\hline \multirow{4}{*}{ 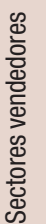 } & \multirow{2}{*}{ Región L } & 1 & $z_{11}^{L L}$ & $z_{12}^{L L}$ & $z_{11}^{L M}$ & $z_{12}^{L M}$ \\
\hline & & 2 & $z_{21}^{L L}$ & $z_{22}^{L L}$ & $z_{21}^{L M}$ & $z_{22}^{L M}$ \\
\hline & \multirow{2}{*}{ Región M } & 1 & $z_{11}^{M L}$ & $z_{12}^{M L}$ & $z_{11}^{M M}$ & $z_{12}^{M M}$ \\
\hline & & 2 & $Z_{21}^{M L}$ & $z_{22}^{M L}$ & $z_{21}^{M M}$ & $z_{22}^{M M}$ \\
\hline
\end{tabular}
muestra en el cuadro 2.

\section{Cuadro 2}

Flujo intersectorial e interregional de bienes

Fuente: Elaboración propia sobre la base de E. R. Miller y P. D. Blair, Input-Output Analysis: Foundations and Extensions, Cambridge, Cambridge University Press, 2009.

Considerando el esquema hipotético del flujo intersectorial e interregional de bienes hacia las regiones $L$ y $M$, ambas con dos sectores, en que $z_{i j}^{L L}$ es el flujo monetario del sector $i$ al sector $j$ en la región $L ; z_{i j}^{M M}$ es el flujo monetario del sector $i$ al sector $j$ en la región $M ; z_{i j}^{L M}$ es el flujo monetario del sector $i$ de la región $L$ al sector $j$ de la región $M ;$ y $z_{i j}^{M L}$ es el flujo monetario del sector $i$ de la región $M$ al sector $j$ de la región $L$.

\section{Metodología}

La metodología utilizada en este artículo se basó en la matriz de insumo-producto, mediante los encadenamientos productivos entre las actividades y la determinación de los sectores clave de la economía, evaluados específicamente mediante los índices de Rasmussen-Hirschman, los campos de influencia y los índices puros de encadenamiento, además de los multiplicadores de producción, empleo e ingreso, que se describen a continuación. 


\section{1. Índice de encadenamiento de Rasmussen-Hirschman}

El índice de encadenamiento desarrollado por Rasmussen (1956) y Hirschman (1958) permite determinar los sectores que tienen mayor poder de encadenamiento dentro de la economía y, por tanto, pueden considerarse sectores clave ${ }^{8}$.

El procedimiento para su estimación parte de la matriz $B$, es decir, la matriz inversa de Leontief, descrita en la ecuación (5.B). Según Guilhoto y otros (2012), el índice de encadenamiento de Rasmussen-Hirschman puede hallarse utilizando las ecuaciones (6) y (7):

$$
\begin{aligned}
& U_{j}=B_{*_{j}} / n / B^{*} \\
& U_{i}=B_{i^{*}} / n / B^{*}
\end{aligned}
$$

donde $U_{j}$ es el índice de encadenamiento hacia atrás y $U_{i}$ corresponde al índice de encadenamiento hacia adelante de Rasmussen-Hirschman, $B$ es la matriz inversa de Leontief; $B^{*}$ es la media de todos los elementos de $B$; y $B_{* j}$ y $B_{i^{*}}$, corresponden, respectivamente, a la suma de cada columna y cada fila de $B$; y $n$ representa el número de sectores de la economía. El índice de encadenamiento hacia atrás indica la medida en que un sector demanda insumos de los otros sectores, mientras el índice de encadenamiento hacia adelante indica la medida en que los productos de un sector son demandados por otros sectores.

Según Haddad (1989), los valores del índice superiores a la unidad pueden denotar sectores clave, que tienen un fuerte encadenamiento con los sectores situados en las fases preliminares y ulteriores de la cadena de producción. Sin embargo, Guilhoto y otros (2012) observan que la aplicación de la metodología del índice de encadenamiento de Rasmussen-Hirschman dificulta la determinación de los coeficientes que, al modificarse, producen un mayor impacto en el sistema en su conjunto. Con miras a cubrir esta laguna, se desarrolló el análisis del campo de influencia.

\section{Campo de influencia}

Según Mendes, Pereira y Teixeira (2011), el enfoque del campo de influencia describe la manera en que los cambios en los coeficientes directos se distribuyen en el sistema económico como un todo y permite verificar las relaciones entre los sectores que presentan mayor relevancia dentro del proceso productivo. Así, el campo de influencia muestra la medida en que cada sector se relaciona hacia atrás y hacia adelante con todos los demás sectores de la economía.

Para llegar a ese resultado, se debe partir de una matriz de coeficientes directos $A=\left|\alpha_{i j}\right|$, definiéndose la matriz de variaciones incrementales en los coeficientes directos de insumo $E=\left|\varepsilon_{i j}\right|$. Las correspondientes matrices de Leontief están dadas por la ecuación (8):

$$
B=[I-A]^{-1}=\left|b_{i j}\right|
$$

y por (9):

$$
B(\varepsilon)=[I-A-\varepsilon]^{-1}=\left|b_{i j}(\varepsilon)\right|
$$

\footnotetext{
8 Véase un abordaje de los sectores clave de la economía también en Hewings y otros (1989).
} 
Si la variación es pequeña y solo se produce en un coeficiente directo, se obtiene:

$$
\varepsilon_{i j}= \begin{cases}\varepsilon, & i=i_{1}, j=j_{1} \\ 0, & i \neq i_{1}, j \neq j_{1}\end{cases}
$$

El campo de influencia de esta variación se puede aproximar mediante la expresión (11):

$$
F\left(\varepsilon_{i j}\right)=\frac{\left[B\left(\varepsilon_{i j}\right)-B\right]}{\varepsilon_{i j}}
$$

donde $F\left(\varepsilon_{i j}\right)$ es una matriz $(n \times n)$ del campo de influencia del coeficiente $\alpha_{i j}$.

Para determinar los coeficientes que tienen mayor campo de influencia, es necesario asociar a cada matriz $F\left(\varepsilon_{i j}\right)$ un valor. Así:

$$
S_{i j}=\sum_{k=1}^{n} \sum_{l=1}^{n}\left[f_{k l}\left(\varepsilon_{i j}\right)\right]^{2}
$$

donde $S_{i j}$ es el valor asociado a la matriz $F\left(\varepsilon_{i j}\right)$. Por lo tanto, los coeficientes directos que presenten los mayores valores de $S_{i j}$ serán aquellos con mayor campo de influencia dentro de la economía en su conjunto.

\section{3. Índices puros de encadenamiento}

De acuerdo con Mendes, Pereira y Teixeira (2011), los índices puros de encadenamiento - que complementan el análisis de la matriz de insumo-producto- determinan el comportamiento de la estructura productiva, considerando el nivel de producción de cada sector y permitiendo dimensionar las interacciones entre sectores en términos de valor de la producción. También conocido como índice GHS, fue propuesto por Guilhoto y otros (1994) con el objetivo de aislar los efectos de cada sector en el sistema económico como un todo9 .

Por lo tanto, considerando la matriz de coeficientes de insumos directos, A (basada en 5.B), que representa un sistema de insumo-producto para un determinado sector j, y el resto de la economía, se obtiene:

$$
A=\left[\begin{array}{ll}
A_{j j} & A_{j r} \\
A_{r j} & A_{r r}
\end{array}\right]
$$

donde $A_{j j}$ y $A_{r r}$ son matrices cuadradas de insumos directos del sector $j$ y del resto de la economía, respectivamente; $A_{j r}$ y $A_{r j}$ son matrices rectangulares que muestran, respectivamente, los insumos directos comprados por el sector $j$ al resto de la economía y los insumos directos comprados por el resto de la economía al sector $j$. Sobre la base de la siguiente matriz inversa de Leontief:

$$
B=(I-A)^{-1}=\left[\begin{array}{ll}
B_{j j} & B_{j r} \\
B_{r j} & B_{r r}
\end{array}\right]=\left[\begin{array}{cc}
\Delta_{j j} & 0 \\
0 & \Delta_{r r}
\end{array}\right]\left[\begin{array}{cc}
\Delta_{j} & 0 \\
0 & \Delta_{r}
\end{array}\right]\left[\begin{array}{cc}
I & A_{j r} \Delta_{r} \\
A_{r j} \Delta_{j} & I
\end{array}\right]
$$

sus elementos se definen como:

$$
\Delta_{j}=\left(I-A_{j j}\right)^{-1}
$$

\footnotetext{
9 En honor a sus creadores, Guilhoto, Hewings y Sonis.
} 


$$
\begin{gathered}
\Delta_{r}=\left(I-A_{r r}\right)^{-1} \\
\Delta_{j j}=\left(I-\Delta_{j} A_{j r} \Delta_{r} A_{r j}\right)^{-1} \\
\Delta_{r r}=\left(I-\Delta r A r j \Delta_{j} A_{j r}\right)^{-1}
\end{gathered}
$$

De este modo, a partir de (14), es posible verificar cómo se produce el proceso de producción en la economía y derivar un conjunto de multiplicadores/encadenamientos representados por las matrices. Al conjugar (15) y (5.B), es posible derivar un conjunto de índices que pueden utilizarse tanto para ordenar los sectores en función de su importancia en el valor de la producción generado, como para verificar la manera en que se produce el proceso de producción en la economía.

A partir de (14) y (5.B), se deduce que:

$$
\left[\begin{array}{l}
X_{j} \\
X_{r}
\end{array}\right]=\left[\begin{array}{cc}
\Delta_{j j} & 0 \\
0 & \Delta_{r r}
\end{array}\right]\left[\begin{array}{cc}
\Delta_{j} & 0 \\
0 & \Delta_{r}
\end{array}\right]\left[\begin{array}{cc}
I & A_{j r} \Delta_{r} \\
A_{r j} \Delta_{j} & I
\end{array}\right]\left[\begin{array}{c}
Y_{j} \\
Y_{r}
\end{array}\right]
$$

de donde es posible derivar las definiciones de índice puro de encadenamiento hacia atrás $(P B L)$ e índice puro de encadenamiento hacia adelante ( $P F L)$, dados respectivamente por (20) y (21):

$$
\begin{aligned}
& P B L=\Delta_{r} A_{r j} \Delta_{j} Y_{j} \\
& P F L=\Delta_{j} A_{j r} \Delta_{r} Y_{r}
\end{aligned}
$$

El $P B L$ proporciona el impacto puro del valor de la producción total del sector $j$ en el resto de la economía, mientras el PFL proporciona el impacto puro del valor de la producción total del resto de la economía en el sector $j$. Como ambos están en valores corrientes, se puede proceder como en la ecuación (22):

$$
P T L=P B L+P F L
$$

Los valores de los índices están normalizados por el valor medio de los sectores de la economía, que permite una comparación, a lo largo del tiempo, en economías que sufren un proceso inflacionario o presentan un patrón monetario modificado. Según Nunes y otros (2012), un sector se considera clave - desde el punto de vista de los índices puros de encadenamiento normalizados - cuando los valores de los índices puros de encadenamiento totales $(P T L)$ normalizados superan la unidad $(P T L>1)$.

\section{Multiplicadores}

Como señalan Tosta, Lirio y Silveira (2012), los multiplicadores de producción, empleo e ingreso suelen utilizarse para cuantificar los efectos de los cambios exógenos en actividades seleccionadas de la economía y pueden clasificarse en multiplicadores de tipo I y de tipo II. La diferencia fundamental entre estos dos tipos es que el segundo modelo considera el consumo de las familias, así como sus respectivas remuneraciones, de forma endógena. 
Básicamente, Miller y Blair (2009) definen los multiplicadores de empleo e ingreso como el incremento del empleo o el salario, respectivamente, dado un choque en la demanda final, que puede representarse matemáticamente mediante la expresión (23):

$$
m(h)_{j}=\sum_{i=1}^{n} \alpha_{n+1} \cdot b_{i j}
$$

donde $m(h)_{j}$ es el multiplicador de empleo (o ingreso) para el sector $j ; \alpha_{n+1}$ está dado por la razón entre el número de personas ocupadas (o el valor agregado a la economía) en el sector y el producto del sector; y $b_{i j}$ es el elemento de la fila $i$ y de la columna $j$ de la matriz inversa de Leontief.

De acuerdo con la definición de Miller y Blair (2009), el multiplicador de producción es el valor total del producto, verificado en toda la economía, necesario para satisfacer una unidad monetaria adicional de demanda final, para la producción procedente de ese sector específico. Matemáticamente, puede expresarse como:

$$
m(o)_{j}=\sum_{i=1}^{n} b_{i j}
$$

donde $m(o)_{j}$ es el multiplicador de producto para el sector $j$; y $b_{i j}$ es el elemento de la fila $i$ y de la columna $j$ de la matriz inversa de Leontief.

Los multiplicadores de tipo II pueden hallarse algebraicamente mediante las ecuaciones presentadas en (23) y (24). Sin embargo, la matriz inversa de Leontief parte de una matriz de coeficientes técnicos en que las familias son endógenos al modelo. En forma esquemática, considerando una economía con solo dos sectores, la matriz A estará dada por:

$$
A=\left[\begin{array}{ccc}
X_{11} / X_{1} & X_{12} / X_{2} & C_{1} / U \\
X_{21} / X_{1} & X_{22} / X_{2} & C_{2} / U \\
V A_{1} / X_{1} & V A_{2} / X_{2} & 0
\end{array}\right]
$$

donde $X_{i j}$ es la producción del sector $i$ para atender al sector $j ; X_{j}$ es la producción total del sector $j$; $C_{i}$ es el consumo privado del sector $i$; $U$ es la suma del consumo privado; y $V A_{j}$ es el valor agregado del sector $j$.

Tomando como endógeno el sector "familias", los multiplicadores de tipo II tienden a minimizar el problema de subestimación que plantean los multiplicadores de tipo I.

\section{Actualización de la matriz de insumo-producto}

El punto de partida para la construcción de la matriz de insumo-producto de la región Nordeste y del resto del Brasil en 2011 fue similar al propuesto por Guilhoto y otros (2012) para 2004. Esta matriz está compuesta por 12 regiones, a saber: los estados que forman parte del área de competencia de la Superintendencia de Desarrollo del Nordeste (SUDENE) (Bahia, Sergipe, Alagoas, Pernambuco, Paraíba, Rio Grande do Norte, Ceará, Piauí, Maranhão, Minas Gerais y Espírito Santo) y el resto del Brasil. En primer lugar, se determinó la matriz de coeficientes técnicos (Matriz A) para 2004, dividiendo el consumo intermedio por el respectivo valor bruto de producción (VBP). A partir de esta, se obtuvo la matriz inversa de Leontief (Matriz $\left.B=(I-A)^{-1}\right)$, de dimensión $1.332 \times 1.332$.

Sobre la base del sistema de cuentas nacionales del Instituto Brasileño de Geografía y Estadística (IBGE, 2014), se midió la variación del valor agregado regional para los estados del Nordeste en el período 2004-2011. Para ello, se consideró la razón entre el volumen del valor agregado bruto de 2004 y el de 2011, teniendo en cuenta los 111 sectores y actividades enumerados en el cuadro 3. 


\section{Cuadro 3}

Brasil: actividades y sectores seleccionados para la matriz de insumo-producto de la región Nordeste, 2011

\begin{tabular}{|c|c|c|c|}
\hline \multicolumn{2}{|c|}{ Sector 0 actividad } & \multicolumn{2}{|c|}{ Sector 0 actividad } \\
\hline 1 & Maíz & 57 & Metalurgia de metales no ferrosos \\
\hline 2 & Caña de azúcar & 58 & Productos de metal, excepto máquinas y equipos \\
\hline 3 & Soja & 59 & Máquinas y utensilios agrícolas \\
\hline 4 & Fruticultura & 60 & $\begin{array}{l}\text { Máquinas y equipos para la prospección } \\
\text { y extracción de petróleo }\end{array}$ \\
\hline 5 & Otros cultivos & 61 & Otras máquinas y equipos \\
\hline 6 & Silvicultura & 62 & Electrodomésticos \\
\hline 7 & Producción de hortalizas & 63 & Máquinas para oficina y equipos de informática \\
\hline 8 & Ganado bovino & 64 & Máquinas, aparatos y materiales eléctricos \\
\hline 9 & Otros animales & 65 & Material electrónico y equipos de comunicaciones \\
\hline 10 & Ganado porcino & 66 & $\begin{array}{l}\text { Aparatos e instrumentos médico- } \\
\text { hospitalarios, de medición y ópticos }\end{array}$ \\
\hline 11 & Aves de corral & 67 & Automóviles, camionetas y vehículos utilitarios \\
\hline 12 & Pescado & 68 & Camiones y ómnibus \\
\hline 13 & Petróleo y otros & 69 & Piezas y accesorios para vehículos automotores \\
\hline 14 & Gas natural & 70 & Otros equipos de transporte \\
\hline 15 & $\begin{array}{l}\text { Actividades de servicio relacionadas con } \\
\text { la extracción de petróleo y gas }\end{array}$ & 71 & Industria del mueble \\
\hline 16 & Mineral de hierro & 72 & Industrias diversas \\
\hline 17 & Otros de la industria extractiva & 73 & Producción de energía eléctrica \\
\hline 18 & Faena de ganado bovino & 74 & Distribución de energía eléctrica \\
\hline 19 & Faena de ganado porcino y otros & 75 & Gas por cañería \\
\hline 20 & Faena de aves & 76 & Agua y alcantarillado \\
\hline 21 & Fabricación de aceites vegetales & 77 & Servicios de limpieza urbana \\
\hline 22 & Industria de productos lácteos & 78 & Construcción \\
\hline 23 & Procesamiento de otros productos vegetales & 79 & Comercio mayorista \\
\hline 24 & Comida de Aves & 80 & Comercio minorista de combustible \\
\hline 25 & Fabricación de azúcar & 81 & Comercio minorista de vehículos, piezas y accesorios \\
\hline 26 & Industria del café & 82 & Supermercados \\
\hline 27 & Otros productos alimenticios & 83 & Otros comercios minoristas \\
\hline 28 & Bebidas & 84 & Transporte de carga por carretera \\
\hline 29 & Productos del tabaco & 85 & Transporte aéreo de carga \\
\hline 30 & Textiles & 86 & Transporte ferroviario de carga \\
\hline 31 & Prendas de vestir y accesorios & 87 & Transporte de carga por agua \\
\hline 32 & Artículos de cuero y calzado & 88 & Transporte de carga por ductos \\
\hline 33 & Productos de madera, excepto muebles & 89 & Actividades auxiliares del transporte de carga \\
\hline 34 & Fabricación de celulosa y pasta mecánica & 90 & Transporte de pasajeros por carretera \\
\hline 35 & Fabricación de papel, cartón y artículos de papel & 91 & Transporte aéreo de pasajeros \\
\hline 36 & Diarios, revistas, discos & 92 & Transporte ferroviario de pasajeros \\
\hline 37 & Refinación de petróleo y coque & 93 & Transporte de pasajeros por agua \\
\hline 38 & Alcohol & 94 & Actividades auxiliares del transporte de pasajeros \\
\hline 39 & Otros elementos químicos & 95 & Correo \\
\hline 40 & Abonos y fertilizantes & 96 & Servicios de telefonía móvil \\
\hline 41 & Fabricación de productos petroquímicos básicos & 97 & Servicios de telefonía fija \\
\hline 42 & Fabricación de productos intermedios para resinas y fibras & 98 & Otros servicios de información \\
\hline 43 & Fabricación de otros productos químicos orgánicos & 99 & Intermediación financiera y seguros \\
\hline 44 & Fabricación de resinas y elastómeros & 100 & Servicios inmobiliarios y de alquiler \\
\hline 45 & Fabricación de fibras, hilos y cables artificiales y sintéticos & 101 & Servicios de mantenimiento y reparación \\
\hline 46 & Farmacia y veterinaria & 102 & Servicios de alojamiento \\
\hline 47 & Pesticidas & 103 & Servicios de alimentación \\
\hline 48 & Perfumería, higiene y limpieza & 104 & Servicios prestados a las empresas \\
\hline 49 & Pinturas, barnices, esmaltes y lacas & 105 & Educación privada \\
\hline
\end{tabular}


Cuadro 3 (conclusión)

\begin{tabular}{llll}
\hline \multicolumn{2}{l}{ Sector o actividad } & Sector o actividad \\
\hline 50 & Productos y preparados químicos diversos & 106 & Salud privada \\
\hline 51 & Industria del caucho & 107 & Otros servicios \\
\hline 52 & Artículos plásticos & 108 & Educación pública \\
\hline 53 & Cemento & 109 & Salud pública \\
\hline 54 & Fabricación de vidrio y productos de vidrio & 110 & Seguridad pública \\
\hline 55 & Otros productos de minerales no metálicos & 111 & Otros servicios de administración pública y seguridad social \\
\hline 56 & Fabricación de acero y derivados & & \\
\hline
\end{tabular}

Fuente: Elaboración propia sobre la base de información oficial.

Los ítems de la demanda final de 2011 se estimaron mediante el producto entre las demandas finales de 2004 y las respectivas razones de crecimiento del valor agregado. La suma de dichos ítems generó un vector columna (1.332x1), que representaba la demanda final para 2011. Al multiplicar la matriz inversa de Leontief (matriz B) por ese vector, se obtiene el VBP de 2011, que también es un vector columna (1.332x1).

Al tomar la matriz de coeficientes técnicos (matriz A) y multiplicarla por el VBP, se obtuvo la matriz del consumo intermedio para 2011 (dimensión 1.332×1.332). A continuación se traspuso el vector columna del VBP, dando origen al vector fila de dimensión 1×1.332, del cual se sustrajo la suma de cada columna del consumo intermedio, que determinó el surgimiento del valor agregado a la producción para 2011. Para la actualización de los valores relativos a los impuestos y la desagregación de los ítems de valor agregado se utilizó la misma proporción registrada en 2004.

Para determinar el ítem "personal ocupado" se midió la variación anual del personal ocupado para cada sector o actividad en el período 2003-2008 (último año disponible), a partir de la cual se proyectó el año 2011.

Después de actualizar la matriz de insumo-producto se realizó la agregación de las 12 regiones en solo 2 regiones, la primera formada por los 9 estados de la región Nordeste y la segunda integrando Minas Gerais y Espírito Santo al resto del Brasil10.

\section{Resultados y análisis}

\section{1. Índices de encadenamiento de Rasmussen-Hirschman}

En el cuadro 4 se observan ocho sectores con fuertes encadenamientos, tanto en las fases preliminares (hacia atrás), como en las ulteriores (hacia adelante) de la cadena productiva, a saber: petróleo y otros (13), textiles (30), refinación de petróleo y coque (37), abonos y fertilizantes (40), fabricación de productos petroquímicos básicos (41), fabricación de resinas y elastómeros (44), fabricación de acero y derivados (56) y productos de metal (excepto máquinas y equipos) (58). A excepción del segundo, todos los sectores y actividades enumerados se caracterizan por pertenecer a la industria de bienes intermedios, lo que les confiere la peculiaridad de tener fuertes niveles de encadenamiento. De este modo, de acuerdo con Prado (1981) y Guilhoto y otros (1994), tales sectores y actividades pueden considerarse piezas clave de la economía del Nordeste en 2011 y definirse como estratégicos para la formulación de políticas sectoriales.

\footnotetext{
10 Para estas etapas se utilizaron los programas informáticos Microsoft $\circledast$ Excel 2013 y MATLAB® R2010a, que permitieron la estimación de las matrices, los coeficientes y los multiplicadores.
} 


\section{Cuadro 4}

Región Nordeste del Brasil: índices de encadenamiento de Rasmussen-Hirschman para 111 sectores seleccionados, 2011

\begin{tabular}{|c|c|c|c|c|c|c|c|c|c|c|c|}
\hline Sector & Adelante & Atrás & Sector & Adelante & Atrás & Sector & Adelante & Atrás & Sector & Adelante & Atrás \\
\hline 1 & 0,72354 & 0,82314 & 29 & 0,53872 & 1,13799 & 57 & 0,76379 & 1,05482 & 85 & 0,66087 & 1,13846 \\
\hline 2 & 1,01153 & 0,70596 & 30 & 1,08843 & 1,08328 & 58 & 1,01705 & 1,03178 & 86 & 0,61709 & 0,97824 \\
\hline 3 & 0,77160 & 0,70299 & 31 & 0,55268 & 1,04472 & 59 & 0,55290 & 1,13785 & 87 & 0,76187 & 0,99129 \\
\hline 4 & 0,67836 & 0,71155 & 32 & 0,61421 & 1,21382 & 60 & 0,54061 & 1,11377 & 88 & 0,70264 & 1,10395 \\
\hline 5 & 1,21008 & 0,77743 & 33 & 0,71347 & 1,03333 & 61 & 0,63537 & 1,11008 & 89 & 1,02975 & 0,91238 \\
\hline 6 & 0,70688 & 0,83189 & 34 & 0,58320 & 1,11122 & 62 & 0,53675 & 1,13188 & 90 & 0,65240 & 0,90732 \\
\hline 7 & 0,63879 & 0,69577 & 35 & 0,81300 & 1,05756 & 63 & 0,55504 & 1,13211 & 91 & 0,63151 & 1,10386 \\
\hline 8 & 1,05394 & 0,85847 & 36 & 0,68633 & 0,96709 & 64 & 0,79273 & 1,08242 & 92 & 0,53382 & 0,89684 \\
\hline 9 & 0,57570 & 0,88255 & 37 & 2,34460 & 1,23225 & 65 & 0,66517 & 1,20464 & 93 & 0,52896 & 0,87796 \\
\hline 10 & 0,74281 & 1,00538 & 38 & 0,60660 & 0,94458 & 66 & 0,56500 & 0,90767 & 94 & 0,62746 & 0,78737 \\
\hline 11 & 0,97111 & 0,91979 & 39 & 0,96093 & 1,16268 & 67 & 0,55683 & 1,32781 & 95 & 0,70868 & 0,78479 \\
\hline 12 & 0,53958 & 0,93099 & 40 & 1,23459 & 1,18894 & 68 & 0,53936 & 1,30662 & 96 & 0,97496 & 0,87398 \\
\hline 13 & 1,02749 & 1,01047 & 41 & 1,10412 & 1,14821 & 69 & 0,88599 & 1,18658 & 97 & 1,05959 & 0,87310 \\
\hline 14 & 0,90663 & 1,01397 & 42 & 0,98196 & 1,28876 & 70 & 0,67221 & 1,19708 & 98 & 1,10018 & 0,82491 \\
\hline 15 & 0,57316 & 0,77573 & 43 & 0,82138 & 1,21740 & 71 & 0,55676 & 1,03816 & 99 & 2,27300 & 0,83964 \\
\hline 16 & 0,53174 & 0,93498 & 44 & 1,41930 & 1,22334 & 72 & 0,57476 & 1,05912 & 100 & 1,02986 & 0,56296 \\
\hline 17 & 0,83825 & 0,95853 & 45 & 0,59717 & 1,11244 & 73 & 1,13878 & 0,64641 & 101 & 0,82337 & 0,71133 \\
\hline 18 & 0,61142 & 1,21165 & 46 & 0,56599 & 0,97388 & 74 & 1,61589 & 0,84980 & 102 & 0,54363 & 0,85866 \\
\hline 19 & 0,56294 & 1,28510 & 47 & 0,87593 & 1,18945 & 75 & 0,91970 & 1,05248 & 103 & 0,68086 & 0,98353 \\
\hline 20 & 0,56295 & 1,23139 & 48 & 0,62772 & 1,08760 & 76 & 0,66747 & 0,69770 & 104 & 2,63910 & 0,77858 \\
\hline 21 & 0,67299 & 1,30239 & 49 & 0,60998 & 1,21366 & 77 & 0,60692 & 0,82046 & 105 & 0,58584 & 0,83967 \\
\hline 22 & 0,58696 & 1,28826 & 50 & 0,68712 & 1,17829 & 78 & 0,77135 & 0,90079 & 106 & 0,57086 & 0,91791 \\
\hline 23 & 0,54419 & 1,17096 & 51 & 0,63465 & 1,12974 & 79 & 3,15717 & 0,69120 & 107 & 0,78992 & 0,80256 \\
\hline 24 & 0,66304 & 1,23598 & 52 & 0,61817 & 1,20879 & 80 & 0,69760 & 0,67856 & 108 & 0,53063 & 0,67256 \\
\hline 25 & 0,70647 & 1,02986 & 53 & 0,62865 & 0,94440 & 81 & 0,66004 & 0,73072 & 109 & 0,52677 & 0,82817 \\
\hline 26 & 0,59904 & 1,29563 & 54 & 0,56697 & 1,00374 & 82 & 0,55883 & 0,71953 & 110 & 0,53168 & 0,82946 \\
\hline 27 & 0,77187 & 1,27484 & 55 & 0,65966 & 1,04157 & 83 & 0,57337 & 0,69741 & 111 & 0,68703 & 0,78170 \\
\hline 28 & 0,72714 & 1,11985 & 56 & 1,03698 & 1,07267 & 84 & 1,86853 & 0,93173 & & & \\
\hline
\end{tabular}

Fuente: Elaboración propia sobre la base de información oficial.

Notas: Los sectores se enumeran en el cuadro 3. Los sectores destacados presentan un índice de Rasmussen-Hirschman hacia atrás o hacia adelante mayor que la unidad.

Cabe destacar que, a pesar de la importancia de la industria petroquímica en el valor de la transformación industrial de la región Nordeste (relevancia destacada por los índices de encadenamiento), Wanderley (2008) afirma que dicho crecimiento no se tradujo en un desarrollo proporcional en algunas industrias que naturalmente se beneficiarían del alto encadenamiento hacia adelante de la industria química. En algunas industrias - como la farmacéutica, la de perfumería y la de plásticos, entre otrasno hubo un incremento significativo. Este comportamiento indica que la materia prima de la industria química no se utilizó en el Nordeste, probablemente debido a la falta de un programa de incentivos para el desarrollo de los sectores que utilizan esta materia prima, que favorezca el encadenamiento de la industria química en la región. Los resultados de la observación del campo de influencia, que se presentan a continuación, ayudan a observar este hecho.

\section{Campo de influencia}

Como complemento del examen de los índices de encadenamiento de Rasmussen-Hirschman, el análisis del campo de influencia evidencia el comportamiento destacado de los sectores clave con respecto a los demás sectores analizados, como se muestra en el cuadro A1.1 del anexo A1. 
La observación del campo de influencia de la economía de la región Nordeste en 2011 corrobora la condición verificada por los índices de encadenamiento hacia atrás y hacia adelante de Rasmussen-Hirschman.

Así, el examen del campo de influencia revela que el sector del petróleo y otros (13) demanda productos y servicios de sectores como otros equipos de transporte (70) y transporte de carga por ductos (88), lo que indica la relevancia de los proveedores de soluciones de transporte para la industria del petróleo.

Con respecto al sector textil (30), se observan numerosos encadenamientos, caracterizados por la demanda de insumos procedentes de los sectores vinculados con las actividades agrícolas (sectores 20, 21, 22, 25 y 26) y la compra de su producción efectuada, entre otros, por el comercio mayorista (79). En este sentido, de acuerdo con Garcia (2010), la configuración de sistemas locales de producción es una característica bastante común en las industrias textil, de prendas de vestir y del calzado en la región Nordeste. En virtud de la simplicidad de la base técnica de estos sectores y de las amplias posibilidades de segmentación del producto, existe un fuerte estímulo al surgimiento y la supervivencia de un vasto conjunto de pequeñas empresas especializadas. Además, la concentración geográfica de las empresas proporciona a los productores un conjunto de beneficios generados por la aglomeración de las empresas y las interacciones entre ellas.

Otro sector importante, el de refinación de petróleo y coque (37), demanda insumos y servicios de sectores como el de transporte de carga por ductos (88) y el de intermediación financiera y seguros (99), mientras tiene encadenamientos hacia atrás con sectores como el textil (30), el de material electrónico y equipos de comunicaciones (65) y la industria de abonos y fertilizantes (40).

La industria de resinas y elastómeros (44) tiene encadenamientos hacia atrás con sectores como el de refinación de petróleo y coque (37), el de transporte de carga por ductos (88) y el de intermediación financiera y seguros (99), y ofrece productos a las industrias textil (30), de material electrónico y equipos de comunicaciones (65) y de productos de madera, excepto muebles (33).

Los encadenamientos mencionados refuerzan la importancia de los sectores considerados clave según el índice de encadenamiento de Rasmussen-Hirschman. Además, demuestran la existencia de básicamente las mismas industrias dominantes en las fases preliminares y ulteriores de la cadena productiva en la economía del Nordeste, evidenciando también la relevancia de los servicios financieros, de transporte y logística y fabricación de equipos electrónicos y de comunicación.

\section{3. Índices puros de encadenamiento}

Al evaluar la economía de la región Nordeste desde el punto de vista de los índices puros de encadenamiento normalizados, o índices GHS, se observa que cinco sectores y actividades presentan un coeficiente total (PTL) superior a la unidad, a saber: refinación de petróleo y coque (37); construcción (78); intermediación financiera y seguros (99); servicios prestados a las empresas (104) y; Otros servicios de administración pública y seguridad social (111) (véase el cuadro 5).

Cabe señalar que, entre los sectores clave determinados según el criterio de Rasmussen-Hirschman, solo el de refinación de petróleo y coque (37) resulta prominente también conforme la metodología GHS. Además, el sector de intermediación financiera y seguros, que ya daba muestras de ser un importante proveedor de servicios, se configura ahora como un sector clave al observar el campo de influencia. Esto lleva a creer que estos sectores son realmente importantes para la economía del Nordeste en 2011.

Algunos sectores no evidenciados por la metodología de Rasmussen-Hirschman aparecen como sectores clave según la metodología de los índices puros de encadenamiento, por ejemplo el sector de Otros servicios de administración pública y seguridad social (111), como también verifican Mendes y otros (2011) al analizar la economía de Minas Gerais. 
Cuadro 5

Región Nordeste del Brasil: índices GHS hacia adelante (PFL), hacia atrás (PBL) y total (PTL) normalizados para 111 sectores seleccionados, 2011

\begin{tabular}{|c|c|c|c|c|c|c|c|c|c|c|c|}
\hline Sector & PFL & $P B L$ & PTL & Sector & PFL & $P B L$ & PTL & Sector & PFL & $P B L$ & PTL \\
\hline 1 & 0,1216 & 0,0710 & 0,0963 & 38 & 0,0886 & 0,0880 & $\begin{array}{l}0,0883 \\
\end{array}$ & 75 & 0,2025 & 0,2763 & 0,2395 \\
\hline 2 & 0,3527 & 0,0282 & 0,1901 & 39 & 0,3190 & 0,0597 & 0,1891 & 76 & 0,1784 & 0,1254 & 0,1518 \\
\hline 3 & 0,2023 & 0,0609 & 0,1315 & 40 & 0,4681 & 0,1198 & 0,2936 & 77 & 0,0920 & 0,0561 & 0,0740 \\
\hline 4 & 0,1224 & 0,0932 & 0,1078 & 41 & 0,4975 & 0,1015 & 0,2991 & 78 & 0,5148 & 3,2660 & 1,8932 \\
\hline 5 & 0,4748 & 0,1844 & 0,3293 & 42 & 0,3734 & 0,1500 & 0,2615 & 79 & 1,3992 & 0,3721 & 0,8846 \\
\hline 6 & 0,0857 & 0,0371 & 0,0613 & 43 & 0,2231 & 0,1011 & 0,1619 & 80 & 0,3940 & 0,0887 & 0,2411 \\
\hline 7 & 0,0526 & 0,0120 & 0,0322 & 44 & 0,7285 & 0,3449 & 0,5363 & 81 & 0,1266 & 0,2286 & 0,1777 \\
\hline 8 & 0,3478 & 0,1511 & 0,2493 & 45 & 0,0241 & 0,0195 & 0,0218 & 82 & 0,0172 & 0,3828 & 0,2004 \\
\hline 9 & 0,0171 & 0,0034 & 0,0102 & 46 & 0,0288 & 0,0344 & 0,0316 & 83 & 0,0281 & 0,4883 & 0,2587 \\
\hline 10 & 0,0663 & 0,0470 & 0,0566 & 47 & 0,2327 & 0,0539 & 0,1431 & 84 & 0,7078 & 0,0406 & 0,3735 \\
\hline 11 & 0,1845 & 0,1249 & 0,1546 & 48 & 0,0866 & 0,1250 & 0,1058 & 85 & 0,0382 & 0,0383 & 0,0383 \\
\hline 12 & 0,0152 & 0,0265 & 0,0209 & 49 & 0,1123 & 0,0240 & 0,0680 & 86 & 0,0288 & $-0,0155$ & 0,0066 \\
\hline 13 & 0,6904 & 0,0417 & 0,3654 & 50 & 0,0800 & 0,0330 & 0,0564 & 87 & 0,1173 & 0,2710 & 0,1943 \\
\hline 14 & 0,3607 & $-0,0750$ & 0,1424 & 51 & 0,0514 & 0,0163 & 0,0338 & 88 & 0,0275 & 0,0079 & 0,0177 \\
\hline 15 & 0,0384 & 0,0026 & 0,0204 & 52 & 0,0262 & 0,0031 & 0,0146 & 89 & 0,2630 & 0,0321 & 0,1473 \\
\hline 16 & 0,0007 & 0,0010 & 0,0009 & 53 & 0,2433 & $-0,0009$ & 0,1210 & 90 & 0,1304 & 0,8949 & 0,5134 \\
\hline 17 & 0,2787 & 0,0596 & 0,1689 & 54 & 0,0400 & 0,0072 & 0,0236 & 91 & 0,1132 & 0,0202 & 0,0666 \\
\hline 18 & 0,0982 & 0,3946 & 0,2467 & 55 & 0,3140 & 0,0346 & 0,1740 & 92 & 0,0064 & 0,0206 & 0,0135 \\
\hline 19 & 0,0112 & 0,0403 & 0,0258 & 56 & 0,2843 & 0,2005 & 0,2423 & 93 & 0,0022 & 0,0012 & 0,0017 \\
\hline 20 & 0,0099 & 0,0817 & 0,0459 & 57 & 0,1678 & 0,2089 & 0,1884 & 94 & 0,0640 & 0,0238 & 0,0438 \\
\hline 21 & 0,1066 & 0,3323 & 0,2197 & 58 & 0,2985 & 0,1618 & 0,2301 & 95 & 0,1362 & 0,0081 & 0,0720 \\
\hline 22 & 0,0233 & 0,0747 & 0,0490 & 59 & 0,0047 & 0,0129 & 0,0088 & 96 & 0,4568 & 0,0641 & 0,2601 \\
\hline 23 & 0,0141 & 0,0503 & 0,0322 & 60 & 0,0008 & 0,0010 & 0,0009 & 97 & 0,4932 & 0,1960 & 0,3443 \\
\hline 24 & 0,1411 & 0,2858 & 0,2136 & 61 & 0,0380 & 0,0960 & 0,0671 & 98 & 0,5755 & $-0,0036$ & 0,2854 \\
\hline 25 & 0,0958 & 0,2941 & 0,1951 & 62 & 0,0022 & 0,0306 & 0,0164 & 99 & 1,7043 & 0,3481 & 1,0248 \\
\hline 26 & 0,0323 & 0,0856 & 0,0590 & 63 & 0,0140 & 0,1125 & 0,0633 & 100 & 0,4912 & 0,1938 & 0,3422 \\
\hline 27 & 0,2345 & 0,6963 & 0,4659 & 64 & 0,1186 & 0,0644 & 0,0914 & 101 & 0,1964 & 0,0613 & 0,1287 \\
\hline 28 & 0,2815 & 0,4787 & 0,3803 & 65 & 0,0101 & 0,0241 & 0,0171 & 102 & 0,0303 & 0,1361 & 0,0833 \\
\hline 29 & 0,0001 & 0,0243 & 0,0122 & 66 & 0,0071 & 0,0204 & 0,0137 & 103 & 0,2442 & 0,9961 & 0,6209 \\
\hline 30 & 0,4939 & 0,3259 & 0,4097 & 67 & 0,0039 & 0,8466 & 0,4261 & 104 & 2,1540 & 0,0491 & 1,0995 \\
\hline 31 & 0,0372 & 0,3856 & 0,2117 & 68 & 0,0014 & 0,0362 & 0,0188 & 105 & 0,0747 & 0,4932 & 0,2844 \\
\hline 32 & 0,0251 & 0,6542 & 0,3403 & 69 & 0,0931 & 0,0268 & 0,0598 & 106 & 0,0393 & 1,0400 & 0,5406 \\
\hline 33 & 0,0458 & 0,0104 & 0,0281 & 70 & 0,0035 & 0,0316 & 0,0176 & 107 & 0,2527 & 1,0419 & 0,6481 \\
\hline 34 & 0,0454 & 0,1020 & 0,0738 & 71 & 0,0254 & 0,1832 & 0,1044 & 108 & 0,0039 & 0,7653 & 0,3854 \\
\hline 35 & 0,1041 & 0,0337 & 0,0688 & 72 & 0,0546 & 0,0479 & 0,0513 & 109 & 0,0001 & 1,2929 & 0,6478 \\
\hline 36 & 0,1430 & 0,0507 & 0,0968 & 73 & 0,6315 & $-0,0273$ & 0,3014 & 110 & 0,0052 & 0,3788 & 0,1923 \\
\hline 37 & 1,3422 & 0,7978 & 1,0694 & 74 & 0,7904 & 0,5608 & 0,6754 & 111 & 0,1209 & 3,9736 & 2,0512 \\
\hline
\end{tabular}

Fuente: Elaboración propia sobre la base de información oficial.

Notas: Los sectores se enumeran en el cuadro 3. Los sectores destacados presentan un índice GHS mayor que la unidad.

\section{Multiplicadores}

Los resultados obtenidos con el método de los multiplicadores, que se muestran a continuación, expresan los efectos en el empleo, los salarios y la producción de una modificación en la demanda final de cada uno de los sectores o actividades seleccionados. Dado que los efectos se propagan a lo largo de toda la cadena, hacia atrás y hacia adelante con respecto al sector objetivo, las industrias con mayores encadenamientos suelen tener los multiplicadores más elevados. Véanse los cuadros A1.1 y A2.1, que contienen los multiplicadores de tipos I y II, respectivamente, en los anexos $A 1$ y $A 2$ de este trabajo. 
Con respecto a los multiplicadores de tipo I, el sector de refinación de petróleo y coque presenta el mayor multiplicador de empleo $(98,19)$ y el segundo mayor multiplicador de salario $(10,84)$, lo que refleja el dinamismo de este sector. Así, por cada 1 millón de reales incrementado en la demanda final de este sector, existe una expectativa de incremento o mantenimiento de 98,19 empleos directos e indirectos y hay una tendencia a que los salarios de la economía respondan positivamente en 10,84 veces al valor del choque inicial. Este resultado concuerda con el obtenido por Nunes, Capucho y Parré (2012) al analizar la economía brasileña, tomando como base el año 2008. Otros sectores, también vinculados a la industria energética, presentaron asimismo resultados significativos en cuanto al aumento del empleo, como en el caso de los sectores de gas por cañería $(80,04)$ y del petróleo y otros $(38,79)$.

Al considerar el multiplicador de producto, cada incremento de 1 millón de reales en la demanda final del sector de automóviles, camionetas y vehículos utilitarios debería provocar un incremento de 2,52 veces ese valor en la producción total de la economía, un valor similar al encontrado por Guilhoto y otros (2012) al analizar la economía de la región Nordeste en 2004. Cabe destacar otros sectores, como la fabricación de aceites vegetales $(2,47)$, la industria del café $(2,46)$, la industria de productos lácteos $(2,45)$ y la faena de ganado porcino y otros $(2,44)$, todos ellos pertenecientes a los agronegocios que, en general, mostraron una buena respuesta a los estímulos sectoriales en la demanda en términos de aumento de la producción.

En el caso de los multiplicadores de tipo II, que se diferencian de los primeros por volver endógeno el sector "gastos de las familias", se observan cambios en la magnitud de los valores de los multiplicadores, así como en el orden de clasificación de los sectores o actividades seleccionados, que pueden dar lugar a sugerencias para formular políticas públicas más específicas y eficaces.

En este sentido, según los resultados obtenidos con el multiplicador de tipo II, el sector de refinación de petróleo y coque continúa presentando el mayor multiplicador de empleo $(118,60)$ y pasa a ser el tercer mayor multiplicador del salario $(12,06)$. Es decir, por cada 1 millón de reales incrementado en la demanda final de este sector, hay una expectativa de incremento o mantenimiento de 118,60 empleos directos, indirectos e inducidos y una tendencia a que los salarios de la economía aumenten 12,06 veces en relación con el valor del choque inicial.

Con respecto al sector del petróleo y otros, se observa un incremento de 48,09 empleos, además de la duplicación del valor de la producción en relación con el choque. Otro sector ligado a la industria del petróleo, el del gas natural, también responde de manera relevante al incremento de la demanda, generando 35,84 empleos directos, indirectos e inducidos adicionales y triplicando con creces el valor del choque en relación con los salarios.

Sabiendo que, en general, las industrias de extracción y refinación de minerales no metálicos y los polos químicos se encuentran en áreas limitadas de la región Nordeste, se presume que estos resultados reflejan, de acuerdo con Lima y Simões (2010), el fortalecimiento de la heterogeneidad dentro de la propia región, donde se observa la coexistencia de áreas estancadas - en las que la modernización, cuando ocurre, es bastante selectiva y limitada- y áreas dinámicas, cuya estructura productiva es bastante moderna y contribuye en gran medida al desempeño de la región en su conjunto ${ }^{11}$.

En general, se observa la prominencia de sectores cuyos insumos son importantes en etapas ulteriores de la cadena de producción, a raíz de los cambios registrados en la economía del Nordeste desde la época del II Plano Nacional de Desenvolvimento (1975-1979). Según Lima y Simões (2010), esto refleja una tendencia a una mayor complementariedad entre los segmentos industriales del Nordeste y del resto del país, especialmente de la región Sudeste, a pesar del traslado

\footnotetext{
${ }^{11}$ Como contrapunto atenuante de esta situación, se puede deducir que hay en curso un relevante movimiento de "internalización del empleo" en la región Nordeste. Según datos del BCB (2006), mientras el 45,9\% de los empleos de la industria estaban dentro de la región en 1996, ese valor ya había aumentado al 51,6\% en 2004.
} 
de industrias productoras de bienes de consumo duraderos al Nordeste, sobre todo a Bahia. Además, la búsqueda de mercados de consumo extrarregionales reiteró la posición de la región Nordeste como proveedora de insumos para las demás regiones del país, concediéndole un lugar peculiar en la división interregional del trabajo industrial en el Brasil.

\section{Consideraciones finales}

El análisis de los resultados obtenidos mediante diferentes metodologías para el examen de la matriz de insumo-producto evidenció la importancia de sectores ya tradicionales para la economía de la región Nordeste. Se destacan la industria textil y los sectores impulsados en el marco del II Plano Nacional de Desenvolvimento (1975-1979), como los sectores químico, de resinas y elastómeros y de petróleo.

Asimismo, mostró la relevancia, aunque incipiente, de sectores como el de material electrónico y equipos de comunicaciones. También se observó que la mayoría de estos sectores depende en gran medida de los sistemas logísticos y los servicios financieros.

En general, dados los encadenamientos observados en varios sectores, se verificó que la economía del Nordeste aún se remonta, en parte, a la organización establecida por las políticas de desarrollo de las décadas de 1970 y 1980. La región sigue desempeñando el papel de centro proveedor de bienes intermedios para las industrias de otras regiones del país, a pesar del reciente traslado de algunas industrias productoras de bienes de consumo duraderos a algunos estados del Nordeste. En consecuencia, su desarrollo no es un proceso autónomo y depende del resto del país.

Sin embargo, cabe notar que para que una política de integración productiva sea eficaz debe basarse en una planificación que incluya infraestructura física de transporte y logística que interconecte las diferentes regiones, además de proyectos nacionales que contemplen la distribución y generación de energía, la capacidad de transmisión de datos y, sobre todo, una educación de calidad.

De este modo, la actuación de los gobiernos estatales y municipales adquiere gran importancia a fin de mitigar las todavía grandes diferencias regionales, por medio de incentivos fiscales y mejoras estructurales, que permitan además la descentralización de la producción, y la dirijan al interior de la región Nordeste, a veces olvidada y carente de sectores productivos más pujantes.

En este sentido, se sugiere a los responsables de la formulación de políticas públicas prestar mayor atención a los incentivos para el sector rural, visto que las actividades de cultivo de caña de azúcar y otros cultivos y la ganadería evidenciaron un fuerte encadenamiento hacia adelante.

Asimismo, se confirmó el intenso encadenamiento hacia atrás de las actividades agroindustriales, que potencia la agregación de valor a los productos, contribuyendo al mantenimiento de los habitantes de las zonas rurales, así como a la mejora del nivel de bienestar, fruto de la conjugación de los efectos multiplicadores del empleo y el salario.

El sector textil, que en el pasado desempeñaba un papel preponderante en la economía de algunos estados de la región Nordeste, también representa una potencial fuente de dinamismo regional, dada su fuerte influencia en las fases preliminares y ulteriores de la cadena productiva. Esto justifica la necesidad de estimularlo mediante políticas públicas específicas. 


\section{Bibliografía}

BCB (Banco Central del Brasil) (2006), "Desconcentração regional da produção da indústria de transformação", Relatório de Inflação [en línea] https://www.bcb.gov.br/content/ri/relatorioinflacao/200609/ RELINF200609-ri200609b1p.pdf.

Delgado, G. C. (1985), Capital financeiro e agricultura no Brasil, 1965-1985, Ícone Editora.

Fishlow, A. (1986), "A economia política do ajustamento brasileiro aos choques do petróleo: uma nota sobre o período 1974/84”, Pesquisa e Planejamento Econômico, vol. 16, № 3.

Galindo, O. (org.) (1997), "Necessidades de infraestrutura econômica do Nordeste. Banco do Nordeste do Brasil. Diretrizes para um Plano de Ação do BNB (1991-1995)", Infraestrutura Econômica e Social do Nordeste, vol. 7, Fortaleza.

Garcia, R. (2010), "Uma análise dos processos recentes de desconcentração regional nas indústrias têxtil e de calçados e a importância dos sistemas locais de produção", Revista Econômica do Nordeste (REN), vol. $41, N^{\circ} 1$.

Gobierno del Brasil (1970), Il Plano Nacional de Desenvolvimento (1975-1979), Brasilia.

Guilhoto, J. J. M. (2007), Análise de insumo-produto: teoria, fundamentos e aplicações, São Paulo, Facultad de Economía, Administración y Contabilidad, Universidad de São Paulo (FEA/USP).

Guilhoto, J. J. y otros (2012), Matriz de insumo-produto do nordeste e estado: metodologia e resultados, Brasilia, Instituto de Investigación Económica Aplicada (IPEA).

Guilhoto, J. J. M y otros (1994), "Índices de ligação e setores-chave da economia Brasileira: 1959/80", Pesquisa e Planejamento Econômico, vol. 24, № 2.

Haddad, P. R. (org.) (1989), Economia Regional: teorias e métodos de análise, Brasilia, Instituto de Investigación Económica Aplicada (IPEA).

Hewings, G. J. y otros (1989), "Key sectors and structural change in the Brazilian economy: a comparsion of alternative approaches and their policy implications", Journal of Policy Modeling, vol. 11, № 1.

Hirschman, A. O. (1958), The Strategy of Economic Development, New Haven, Yale University Press.

IBGE (Instituto Brasileño de Geografía y Estadística) (2014), "Sistema de Contas Nacionais" [en línea] http:// www.ibge.gov.br.

Leontief, W. W. (1966), The Structure of American Economy: 1919-1939, Nueva York, Oxford University.

Lessa, C. (1977), "Visão crítica do II PND", Revista Tibiriçá, vol. 2, № 6.

Lima, A. C. da C. y R. F. Simões (2010), "Centralidade e emprego na região Nordeste do Brasil no período 1995/2007", Nova Economia, vol. 20, № 1.

Mendes, C. S., M. W. G. Pereira y E. C. Teixeira (2011), "Uma análise do insumo-produto do setor lácteo mineiro", Revista Econômica do Nordeste (REN), Fortaleza-CE, vol. 42, № 03, julio/septiembre.

Miller, R. (1998), "Regional and interregional input-output analysis", Methods of Interregional and Regional Analysis, I. Aziz y otros, Aldershot, Ashgate.

Miller, R. y P. D. Blair (2009), Input-Output Analysis: Foundations and Extensions, Cambridge, Cambridge University Press.

Montoya, M. A., E. B. Finamore y C. A. Pasqual (2012), "Fontes de crescimento e mudança estrutural na economia gaúcha: uma análise do Valor Bruto da Produção (VBP) e do emprego", Ensaios FEE, vol. 33, № 2, noviembre.

Morrone, H. (2017), "Estimando os impactos do aumento do ICMS na economia gaúcha: uma análise de insumo-produto", Revista Economia Ensaios, vol. 31, № 2, Uberlândia, Minas Gerais, enero/junio.

Nunes, P. A., T. O. Capucho y J. L. Parré (2012), "Estrutura produtiva Brasileira e Paranaense: comparações sobre os índices de ligação (R-H e GHS) e os multiplicadores de produção, renda e emprego", Revista de Desenvolvimento Econômico (RDE), Salvador, año XIV, № 25, junio.

Prado, E. F. S. (1981), Estrutura Tecnológica de Desenvolvimento Regional, São Paulo, Universidad de São Paulo.

Rasmussen, P. (1956), Studies in Intersectoral Relations, Amsterdam, North-Holland.

Ribeiro, L. C. S. y A. P. V. Leite (2012), "Estrutura econômica do Estado de Sergipe em 2006: uma contribuição através da matriz de insumo-produto", Revista Econômica do Nordeste (REN), vol. 43, № 04, Fortaleza, octubre-diciembre.

Ribeiro, L. C. S. y otros (2013), "Suape: um novo polo de crescimento?", Novos Cadernos NAEA, vol. 16, № 1 , junio.

Tosta, M. C. R., V. S. Lirio y S. F. R. Silveira (2004), "Matrizes de insumo-produto: construção, uso e aplicações", Métodos Quantitativos em Economia, M. L. dos Santos y W. C. Vieira (eds.), Viçosa, Universidad Federal de Viçosa.

Wanderley, L. A. (2008), "Integração nacional e fragmentação regional da indústria de transformação: Sudeste e Nordeste", Revista Economia Política do Desenvolvimento, vol. 1, № 3. 


\title{
Anexo A1
}

\section{Multiplicadores de producción, empleo e ingreso de tipo I en la región Nordeste, 2011}

\author{
Cuadro A1.1
}

Región Nordeste del Brasil: multiplicadores de producción, empleo e ingreso de tipo I, 2011

\begin{tabular}{|c|c|c|c|c|c|c|c|c|c|c|c|}
\hline \multirow{2}{*}{ Sectores } & \multicolumn{3}{|c|}{ Multiplicadores I } & \multirow{2}{*}{ Sectores } & \multicolumn{3}{|c|}{ Multiplicadores I } & \multirow{2}{*}{ Sectores } & \multicolumn{3}{|c|}{ Multiplicadores I } \\
\hline & Empleo & Ingreso & Producción & & Empleo & Ingreso & Producción & & Empleo & Ingreso & Producción \\
\hline 1 & 1,0610 & 1,2306 & 1,5629 & 38 & 20,1414 & 3,8610 & 1,7934 & 75 & 80,0401 & 10,7379 & 1,9983 \\
\hline 2 & 1,0152 & 1,0814 & 1,3404 & 39 & 10,4201 & 3,6488 & 2,2075 & 76 & 1,9771 & 1,2401 & 1,3247 \\
\hline 3 & 1,3704 & 1,6294 & 1,3347 & 40 & 9,2598 & 3,3028 & 2,2574 & 77 & 1,3147 & 1,2304 & 1,5578 \\
\hline 4 & 1,0153 & 1,1703 & 1,3510 & 41 & 7,4355 & 2,7333 & 2,1801 & 78 & 1,3303 & 1,8623 & 1,7103 \\
\hline 5 & 1,1324 & 1,5127 & 1,4761 & 42 & 6,9810 & 15,4360 & 2,4469 & 79 & 1,3066 & 1,1933 & 1,3123 \\
\hline 6 & 1,1931 & 1,4597 & 1,5795 & 43 & 6,3801 & 5,8975 & 2,3114 & 80 & 1,2635 & 1,1641 & 1,2884 \\
\hline 7 & 1,0890 & 1,1988 & 1,3210 & 44 & 18,9709 & 3,9483 & 2,3227 & 81 & 1,1721 & 1,2354 & 1,3874 \\
\hline 8 & 1,1212 & 1,3583 & 1,6299 & 45 & 2,7822 & 2,3543 & 2,1121 & 82 & 1,1487 & 1,2193 & 1,3661 \\
\hline 9 & 1,0599 & 1,4378 & 1,6757 & 46 & 3,4064 & 1,8521 & 1,8491 & 83 & 1,0673 & 1,1894 & 1,3241 \\
\hline 10 & 1,8175 & 1,8438 & 1,9089 & 47 & 19,7539 & 4,5913 & 2,2584 & 84 & 1,4524 & 1,8784 & 1,7690 \\
\hline 11 & 1,1983 & 1,5198 & 1,7464 & 48 & 4,0184 & 2,0827 & 2,0650 & 85 & 10,7877 & 3,8855 & 2,1615 \\
\hline 12 & 1,0662 & 1,3687 & 1,7676 & 49 & 7,9493 & 2,3100 & 2,3043 & 86 & 1,8228 & 1,4757 & 1,8573 \\
\hline 13 & 38,7929 & 2,6379 & 1,9185 & 50 & 2,9262 & 2,3720 & 2,2372 & 87 & 3,6958 & 2,1877 & 1,8821 \\
\hline 14 & 29,3068 & 3,0267 & 1,9252 & 51 & 5,0258 & 2,1699 & 2,1450 & 88 & 2,5914 & 2,5286 & 2,0960 \\
\hline 15 & 2,4112 & 1,2021 & 1,4728 & 52 & 1,9191 & 1,8454 & 2,2951 & 89 & 1,4143 & 1,3997 & 1,7323 \\
\hline 16 & 8,9887 & 3,6018 & 1,7752 & 53 & 8,8002 & 2,2286 & 1,7931 & 90 & 1,2403 & 1,3733 & 1,7227 \\
\hline 17 & 1,5695 & 1,7090 & 1,8199 & 54 & 2,5234 & 2,0642 & 1,9058 & 91 & 6,6024 & 2,1911 & 2,0959 \\
\hline 18 & 29,7316 & 5,0114 & 2,3005 & 55 & 1,4927 & 1,7492 & 1,9776 & 92 & 1,7023 & 1,2517 & 1,7028 \\
\hline 19 & 13,4425 & 3,8766 & 2,4400 & 56 & 7,2917 & 2,9059 & 2,0366 & 93 & 1,9280 & 1,3035 & 1,6669 \\
\hline 20 & 14,5036 & 4,1864 & 2,3380 & 57 & 2,8568 & 2,7634 & 2,0027 & 94 & 1,3505 & 1,2514 & 1,4950 \\
\hline 21 & 20,3202 & 7,3520 & 2,4728 & 58 & 1,7774 & 1,9088 & 1,9590 & 95 & 1,3098 & 1,1664 & 1,4900 \\
\hline 22 & 7,3290 & 3,8428 & 2,4460 & 59 & 2,7529 & 2,1207 & 2,1604 & 96 & 1,4637 & 2,0419 & 1,6594 \\
\hline 23 & 8,0674 & 3,3269 & 2,2232 & 60 & 1,5288 & 1,7546 & 2,1147 & 97 & 2,3643 & 2,4961 & 1,6577 \\
\hline 24 & 7,4981 & 2,9379 & 2,3467 & 61 & 2,0609 & 2,0218 & 2,1077 & 98 & 1,6343 & 1,4870 & 1,5662 \\
\hline 25 & 12,3703 & 3,1589 & 1,9554 & 62 & 2,3880 & 2,2009 & 2,1491 & 99 & 3,2768 & 1,5778 & 1,5942 \\
\hline 26 & 10,5345 & 3,6901 & 2,4600 & 63 & 6,1804 & 2,5824 & 2,1495 & 100 & 3,9215 & 1,5765 & 1,0689 \\
\hline 27 & 3,9344 & 3,0116 & 2,4205 & 64 & 2,9882 & 1,7897 & 2,0551 & 101 & 1,2768 & 1,1945 & 1,3506 \\
\hline 28 & 6,6452 & 2,5066 & 2,1262 & 65 & 4,6439 & 3,8798 & 2,2872 & 102 & 2,4056 & 1,2308 & 1,6303 \\
\hline 29 & 13,3877 & 2,9401 & 2,1607 & 66 & 1,4977 & 1,6080 & 1,7233 & 103 & 1,3821 & 1,7169 & 1,8674 \\
\hline 30 & 1,8717 & 2,3792 & 2,0568 & 67 & 12,1553 & 3,4727 & 2,5211 & 104 & 1,2100 & 1,3152 & 1,4783 \\
\hline 31 & 1,2523 & 1,7580 & 1,9836 & 68 & 7,2150 & 4,2123 & 2,4808 & 105 & 1,3341 & 1,2027 & 1,5942 \\
\hline 32 & 2,2513 & 2,1879 & 2,3046 & 69 & 3,0403 & 2,1045 & 2,2529 & 106 & 1,4245 & 1,4321 & 1,7428 \\
\hline 33 & 1,8550 & 1,8151 & 1,9619 & 70 & 3,4722 & 2,3190 & 2,2728 & 107 & 1,0770 & 1,1660 & 1,5238 \\
\hline 34 & 28,9656 & 4,5228 & 2,1098 & 71 & 1,8252 & 2,1092 & 1,9711 & 108 & 1,1564 & 1,0619 & 1,2770 \\
\hline 35 & 2,9515 & 1,8823 & 2,0079 & 72 & 1,6153 & 2,1821 & 2,0109 & 109 & 1,7604 & 1,2256 & 1,5724 \\
\hline 36 & 1,7622 & 1,5742 & 1,8362 & 73 & 7,3128 & 1,8755 & 1,2273 & 110 & 1,8203 & 1,2825 & 1,5749 \\
\hline 37 & 98,1900 & 10,8380 & 2,3396 & 74 & 4,8449 & 1,9481 & 1,6135 & 111 & 1,4923 & 1,1939 & 1,4842 \\
\hline
\end{tabular}

Fuente: Elaboración propia sobre la base de información oficial.

Nota: Los sectores se enumeran en el cuadro 3. 


\section{Anexo A2}

\section{Multiplicadores de producción, empleo e ingreso de tipo II en la región Nordeste, 2011}

Cuadro A2.1

Región Nordeste del Brasil: multiplicadores de producción, empleo e ingreso de tipo II, 2011

\begin{tabular}{|c|c|c|c|c|c|c|c|c|c|c|c|}
\hline \multirow{2}{*}{ Sectores } & \multicolumn{3}{|c|}{ Multiplicadores II } & \multirow{2}{*}{ Sectores } & \multicolumn{3}{|c|}{ Multiplicadores II } & \multirow{2}{*}{ Sectores } & \multicolumn{3}{|c|}{ Multiplicadores II } \\
\hline & Empleo & Ingreso & Producción & & Empleo & Ingreso & Producción & & Empleo & Ingreso & Producción \\
\hline 1 & 1,1031 & 1,4281 & 1,8964 & 38 & 20,7981 & 4,2940 & 2,0138 & 75 & 96,0721 & 12,0887 & 2,1168 \\
\hline 2 & 1,0380 & 1,2069 & 1,7198 & 39 & 12,9557 & 4,0598 & 2,3331 & 76 & 2,6151 & 1,3628 & 1,4687 \\
\hline 3 & 2,0700 & 2,6087 & 1,6598 & 40 & 11,6067 & 3,6677 & 2,3968 & 77 & 1,5437 & 1,3517 & 1,8023 \\
\hline 4 & 1,0372 & 1,4159 & 1,6968 & 41 & 9,2919 & 3,0377 & 2,3013 & 78 & 1,4981 & 2,1696 & 1,9193 \\
\hline 5 & 1,2197 & 2,0039 & 1,7925 & 42 & 8,3087 & 17,2399 & 2,5509 & 79 & 1,6144 & 1,3684 & 1,5929 \\
\hline 6 & 1,2858 & 1,8181 & 1,8985 & 43 & 7,6214 & 6,5786 & 2,4228 & 80 & 1,5483 & 1,3281 & 1,5782 \\
\hline 7 & 1,1493 & 1,4810 & 1,6802 & 44 & 23,7181 & 4,3913 & 2,4276 & 81 & 1,3091 & 1,4066 & 1,6692 \\
\hline 8 & 1,1884 & 1,6499 & 2,0388 & 45 & 3,3501 & 2,6098 & 2,2356 & 82 & 1,2652 & 1,3857 & 1,6540 \\
\hline 9 & 1,0829 & 1,7474 & 2,0666 & 46 & 4,1063 & 2,0511 & 2,0332 & 83 & 1,1190 & 1,3558 & 1,6105 \\
\hline 10 & 2,0871 & 2,3044 & 2,2885 & 47 & 23,1338 & 5,1088 & 2,3870 & 84 & 1,6580 & 2,2064 & 1,9986 \\
\hline 11 & 1,2572 & 1,8485 & 2,1454 & 48 & 4,7394 & 2,3201 & 2,2419 & 85 & 14,3167 & 4,5096 & 2,3513 \\
\hline 12 & 1,0877 & 1,5989 & 2,1533 & 49 & 9,9259 & 2,5665 & 2,4716 & 86 & 2,2710 & 1,6352 & 2,1122 \\
\hline 13 & 48,0909 & 2,9363 & 2,0709 & 50 & 3,4319 & 2,6365 & 2,3951 & 87 & 4,9078 & 2,5741 & 2,0924 \\
\hline 14 & 35,8448 & 3,3757 & 2,0681 & 51 & 5,9869 & 2,4216 & 2,3004 & 88 & 3,1631 & 2,9275 & 2,3414 \\
\hline 15 & 3,4865 & 1,3211 & 1,7277 & 52 & 2,2801 & 2,0379 & 2,4691 & 89 & 1,6870 & 1,5853 & 1,9922 \\
\hline 16 & 10,7869 & 4,0221 & 1,8721 & 53 & 11,1774 & 2,4754 & 1,9165 & 90 & 1,3962 & 1,5533 & 1,9823 \\
\hline 17 & 1,7934 & 1,9048 & 1,9892 & 54 & 3,0424 & 2,3055 & 2,0688 & 91 & 8,9529 & 2,4878 & 2,3055 \\
\hline 18 & 32,0626 & 5,8864 & 2,5955 & 55 & 1,6735 & 1,9417 & 2,1633 & 92 & 2,3063 & 1,3778 & 2,0225 \\
\hline 19 & 14,8433 & 4,5699 & 2,7225 & 56 & 9,1601 & 3,2282 & 2,1518 & 93 & 2,6736 & 1,4745 & 1,9247 \\
\hline 20 & 15,4027 & 4,9165 & 2,6401 & 57 & 3,4338 & 3,0664 & 2,1190 & 94 & 1,6547 & 1,4224 & 1,7842 \\
\hline 21 & 25,1616 & 9,2273 & 2,7217 & 58 & 2,1457 & 2,1389 & 2,1160 & 95 & 1,5923 & 1,2845 & 1,7606 \\
\hline 22 & 7,9000 & 4,4391 & 2,7384 & 59 & 3,4318 & 2,3473 & 2,3305 & 96 & 1,5941 & 2,3183 & 1,8260 \\
\hline 23 & 8,5199 & 3,8530 & 2,4861 & 60 & 1,7645 & 1,9375 & 2,3115 & 97 & 2,7690 & 2,8616 & 1,8098 \\
\hline 24 & 8,3630 & 3,4324 & 2,5930 & 61 & 2,4770 & 2,2389 & 2,2771 & 98 & 1,9188 & 1,6591 & 1,7876 \\
\hline 25 & 12,8413 & 3,5054 & 2,1962 & 62 & 2,8603 & 2,4351 & 2,3177 & 99 & 4,2172 & 1,7431 & 1,7812 \\
\hline 26 & 11,6323 & 4,3496 & 2,7230 & 63 & 7,8418 & 2,8664 & 2,3170 & 100 & 5,0929 & 1,8217 & 1,0949 \\
\hline 27 & 4,3260 & 3,4869 & 2,6616 & 64 & 3,8362 & 1,9764 & 2,2404 & 101 & 1,8652 & 1,4305 & 1,7033 \\
\hline 28 & 7,3866 & 2,8288 & 2,3561 & 65 & 5,5107 & 4,3179 & 2,4430 & 102 & 3,1556 & 1,3716 & 1,9602 \\
\hline 29 & 14,8288 & 3,4461 & 2,3885 & 66 & 1,7524 & 1,7939 & 1,9051 & 103 & 1,4869 & 1,9871 & 2,1509 \\
\hline 30 & 2,0364 & 2,7289 & 2,2506 & 67 & 15,3430 & 3,8560 & 2,7148 & 104 & 1,3612 & 1,4971 & 1,7556 \\
\hline 31 & 1,3295 & 2,0627 & 2,2846 & 68 & 8,8601 & 4,6785 & 2,6583 & 105 & 1,5472 & 1,3285 & 1,9444 \\
\hline 32 & 2,5449 & 2,4451 & 2,5416 & 69 & 3,7748 & 2,3253 & 2,4425 & 106 & 1,6282 & 1,6146 & 2,0546 \\
\hline 33 & 2,0143 & 2,0349 & 2,1885 & 70 & 4,2979 & 2,5663 & 2,4561 & 107 & 1,1352 & 1,2927 & 1,8951 \\
\hline 34 & 32,1407 & 5,2212 & 2,2836 & 71 & 2,0814 & 2,4000 & 2,1692 & 108 & 1,4260 & 1,1630 & 1,6985 \\
\hline 35 & 3,5056 & 2,0891 & 2,1832 & 72 & 1,8450 & 2,4882 & 2,1976 & 109 & 2,1977 & 1,3474 & 1,9118 \\
\hline 36 & 2,1069 & 1,7476 & 2,0505 & 73 & 8,9747 & 2,0736 & 1,2748 & 110 & 2,3155 & 1,4096 & 1,8322 \\
\hline 37 & 118,5966 & 12,0601 & 2,4499 & 74 & 6,2108 & 2,1490 & 1,6845 & 111 & 1,8822 & 1,3104 & 1,7816 \\
\hline
\end{tabular}

Fuente: Elaboración propia sobre la base de información oficial.

Nota: Los sectores se enumeran en el cuadro 3. 